\title{
Relacionando la legislación sobre acceso a la Información de los países del MERCOSUR
}

\author{
Terezinha Elisabeth da Silva \\ Thiago Gomes Eirão \\ Raphael da Silva Cavalcante \\ Câmara dos Deputados, Brasil
}

\section{REVIEW}

\begin{abstract}
Resumo
Esta investigación tuvo como corpus las Leyes de Acceso a la Información de los países miembros del MERCOSUR, proponiendo verificar la existencia de la relación entre las Leyes descritas. Basándose en el texto de la Ley de Acceso a la Información, (Argentina, Brasil, Chile, Colombia, Ecuador, Perú y Uruguay), de los proyectos de ley (Paraguay y Bolivia) y de la Constitución (Venezuela), se estudiaron a partir de tres puntos de vista: transparencia activa, transparencia pasiva y sigilo. Este punto de partida permite identificar signos de una agenda común acerca del tema entre los países que pertenecen al bloque. Los resultados indican semejanzas entre las Leyes, siendo que en los tres aspectos estudiados se encuentran resonancia en mayor o menor grado.
\end{abstract}

Palabras-clave

Acceso a la información ; Información pública ; MERCOSUR ; Legislacion ; Transparencia ; Secreto burocrático ; Sigilo ; Argentina ; Bolivia ; Brasil ; Chile ; Colombia ; Ecuador ; Paraguay ; Perú ; Uruguay ; Venezuela

\section{Linking the legislation about information acces in countries from MERCOSUR}

\begin{abstract}
This research had as core the information access laws from countries members of MERCOSUL. The purpose was to verify the existence of relationship between these laws. Information access laws (Argentina, Brazil, Chile, Colombia, Ecuador, Peru and Uruguay), Bills (Paraguay and Bolivia) and a Constitution (Venezuela) were studies from three approaches: active transparency, passive transparency and secrecy. These starts allow identify signals of a common agenda around the theme among the countries members. The results shows similarities between the laws and the three aspects studied find resonance in bigger or smaller degree.
\end{abstract}

Keywords

Freedom of information; Access to information ; MERCOSUR ; Law ; Transparency ; Bureaucratic secrecy ; Secrecy; Argentina ; Bolivia ; Brazil ; Chile ; Colombia ; Ecuador ; Paraguay ; Peru ; Uruguay ; Venezuela

\section{Introducción}

Firmado en 1991 por Argentina, Brasil, Paraguay y Uruguay, el Tratado de Asunción representó el primer paso concreto para la construcción de un área de integración de los países signatarios. Fruto del histórico de las relaciones mantenidas por esos países, surgía así la instancia formal de interacción, el Mercado Común del Sur (MERCOSUR). Con la intención de crear, en un primer momento, un espacio de libre circulación de bienes y servicios, la formación de ese bloque también significó la posibilidad de pensar en los países sudamericanos como entidades interconectadas.

Unir las agendas de esos países indicaba una respuesta al bloque europeo que se fortalecía y convergía para la total integración económica, política y social. Veinte y tres años después de su creación, el MERCOSUR ha pasado por algunas modificaciones tanto en la cantidad de países que conforman el bloque, como en las orientaciones de 
sus actividades, vislumbrando la posibilidad de acciones más allá del plan económico. Entre los integrantes y asociados, actualmente el MERCOSUR está formado por diez países.

Ese contexto proporciona un punto de partida que permite cuestionar si es posible encontrar en el MERCOSUR puntos de relación y de convergencia de actuación de los estados participantes además de las políticas económicas. Teniendo como corpus de estudio las Leyes de Acceso a la Información de los países miembros, esta investigación se propone verificar la existencia de la relación entre las Leyes referidas en los países integrantes y asociados del MERCOSUR. Tal abordaje permite identificar signos de una agenda común acerca del tema entre los países que pertenecen al bloque.

\section{Leyes de acceso a la información en los paises del MERCOSUR}

Argentina, Brasil, Chile, Colombia, Ecuador, Perú y Uruguay ya tienen Leyes de Acceso a la Información. En Paraguay se tramita un proyecto de ley y en Bolivia existe una minuta de ley en discusión. En Venezuela no hay una ley específica o proyecto siendo tramitado en las Casas legislativas acerca de la materia, siendo que la Constitución venezolana trata el asunto de manera periférica y sucinta.

Los documentos relativos a las Leyes de Acceso a la Información, sean las normas propiamente dichas, el proyecto de ley paraguayo, la minuta de ley boliviana y la Constitución venezolana fueron estudiadas a partir de tres puntos de vista: transparencia activa, transparencia pasiva y sigilo. Esos aspectos son considerados orientadores de los diplomas legales que rigen el acceso a la información pública de los Estados y tienen como base las directrices emanadas de la Organización de las Naciones Unidas y de la Organización de los Estados Americanos.

El cuadro 1 presenta la lista de los países con sus respectivas normas y como trataron los tres aspectos abordados en este trabajo:

Cuadro 1: Países del MERCOSUR y las leyes de acceso a la información

\begin{tabular}{|c|c|c|c|c|c|}
\hline País & Ley & Año & Sigilo & Transparencia Activa & Transparencia Pasiva \\
\hline Argentina & Decreto 1172 (Anexo VII), & 2003 & Si, art. 16 & Sí. art. 10 & Sí. Arts. 11 a 17 \\
\hline Bolivia & Minuta de proyecto de ley & 2013 & $\mathrm{Si}$, art 42 e 43 & Si, art. 6 e 8 & Si, art. 25 \\
\hline Brasil & 12527 & 2011 & Si, art. 23 e 24 & $\mathrm{Si}$, art. $8^{\circ}$ & Si, art. 10 \\
\hline Chile & 20285 & 2009 & Si, art. 21 a 26 & Si, art. 7 & Si, art. 10 \\
\hline Colombia & 1712 & 2014 & Si art. 18 e 19 & Si, art. 7 a 11 & Si, art. 24 \\
\hline Ecuador & 27806 & 2004 & si art. 17 e 18 & $\mathrm{Si}$, art 7 e 8 & Si, art. 19 \\
\hline Paraguay & Proyecto de ley S-136271 & 2013 & No & $\mathrm{Si}$, art. 9. & Si, art. 13 \\
\hline Perú & 27806 & 2002 & Si art. 15/15-A/15-B & Si, art. 3 e 5 & Si, art. 7 a 11 \\
\hline Uruguay & 18.831 & 2008 & Sí. art. 8 a 12 & Sí. art. 5 & Sí. Arts. 13 a 18 \\
\hline Venezuela & No tiene ley & - & - & - & - \\
\hline
\end{tabular}

Fuente: elaboración de los autores.

\section{a) Argentina}

Así como ocurrió con muchos países de América del Sur que fueron gobernados por dictaduras militares en la segunda mitad del siglo XX, la reforma constitucional argentina post dictadura, realizada en 1994, reveló en el texto de la nueva Carta la necesidad de garantizar a los argentinos parte de los derechos que vienen siendo establecidos por las Leyes de Acceso a la Información contemporánea. 
Sin embargo, sólo en 2003 se promulgó una legislación específica a nivel nacional que regulara de forma objetiva el derecho de acceso a la información. Se trata del Reglamento General del Acceso a la Información Pública para el Poder Ejecutivo Nacional, presentado por el Decreto 1.172/2003, dirigido únicamente a instituciones públicas integrantes del Poder Ejecutivo. El Reglamento se basa en cinco principios: igualdad, publicidad, celeridad, informalidad y gratuidad, teniendo como finalidad "permitir y promover una efectiva participación ciudadana, a través de la provisión de información completa, adecuada, oportuna y veraz" (ARGENTINA, 2003).

Actualmente, en Argentina todavía no existe una ley de ámbito nacional sobre el acceso a la información que pase todos los poderes del Estado, aunque estén en trámite en el Congreso Nacional 22 proyectos de ley sobre del tema.

\section{b) Brasil}

El marco fundamental de la Ley 12.257/2011 es la Constitución de 1988 que apunta, en el inciso XXXIII del artículo $5^{\circ}$, el derecho de acceso a la información:

XXXIII - todos tienen derecho a recibir de los organismos públicos informaciones de su interés particular, o de interés colectivo o general, que serán dadas en el plazo de la ley, bajo pena de responsabilidad, excepto aquellas cuyo sigilo sea imprescindible para la seguridad de la sociedad y del Estado;

La Ley de Acceso a la Información (LAI), resulta del Proyecto de Ley 219/2003 que tuvo la finalidad de determinar el marco regulatorio de las relaciones entre Estado y sociedad, garantizando un amplio acceso a las informaciones. Después de casi 10 años de tramitación y discusión entre Estado y sociedad civil la ley empezó a producir efectos en 2012.

Fueron largos años de lucha hasta que la Constitución (1988), el proyecto de Ley (2003) y la Ley (2011), concretaron las acciones de democratización iniciadas inmediatamente después del fin de la dictadura militar en 1985. La referida ley representó para la sociedad, una nueva forma de relación con el Estado que pasó a implementar un nuevo modelo de sigilo de la información, ya que el principio de la transparencia máxima y el acceso son las bases de la nueva ley. Dentro del MERCOSUL Brasil es uno de los países que más tarde reglamentó el acceso a la información, en consecuencia, todavía sigue adaptándose a la nueva realidad.

\section{c) Chile}

El 4 de enero de 2005 dos senadores presentaron el proyecto de ley ${ }^{1}$ que cuatro años más tarde se convertiría en la ley no 20.285/2009², la ley de acceso a la información chilena. Según Larraín Fernández y Gazmuri Mujica (2005, p. 1)

El derecho al libre acceso a las fuentes públicas de información, entendido como la posibilidad real de la ciudadanía de tomar conocimiento de los actos de la administración del estado y de la documentación que sustenta tales actos, es un tema relativamente nuevo en nuestro país.Este derecho constituye un elemento fundamental para alcanzar un alto grado de transparencia en el ejercicio de las funciones públicas, a la vez que facilita la formación de una mayor y más efectiva participación ciudadana en los asuntos públicos.

La referida ley colocó a Chile en el grupo de los países del mundo que crearon y aseguraron las condiciones del amplio acceso a las informaciones públicas. La fuerza motivadora para la creación de la ley se puede encontrar en el artículo $8^{\circ}$ de la Constitución del país que lo declaró:

Artículo $8^{\circ}$.- El ejercicio de las funciones públicas obliga a sus titulares a dar estricto cumplimiento al principio de probidad en todas sus actuaciones. Son públicos los actos y resoluciones de los órganos del Estado, así como sus fundamentos y los procedimientos que utilicen. Sin embargo, sólo una ley de quórum calificado podrá establecer la reserva o secreto de aquellos o de éstos, cuando la publicidad afectare el debido cumplimiento de las funciones de dichos órganos, los derechos de las personas, la seguridad de la Nación o el interés nacional. (CHILE. CONSTITUCIÓN, 1980) 


\section{d) Paraguay}

A pesar de no tener una ley de acceso a la información, en 1992 Paraguay trató del asunto en su Constitución al declarar en su artículo 28:

Se reconoce el derecho de las personas a recibir información veraz, responsable y ecuánime. Las fuentes públicas de información son libres para todos. La ley regulará las modalidades, plazos y sanciones correspondientes a las mismas, a fin de que este derecho sea efectivo.

Aunque de forma sucinta, la Carta Magna paraguaya estableció los primeros principios básicos para promover el acceso a la información. Veintiún años después se presentó el proyecto de ley S-136271 ${ }^{3}$ para reglamentar el referido artículo y garantizar el libre acceso del ciudadano a la información pública, garantizando de esa forma la transparencia gubernamental.

Después del primer trámite constitucional en el Senado, el proyecto pasó a la Cámara de los Diputados que en junio de 2014 devolvió el proyecto al senado para el tercer trámite constitucional. Fruto de muchas discusiones en el campo político, el proyecto todavía camina a pasos lentos para su concretización en ley.

En cuanto a la estructura del proyecto, actualmente el texto está en trámite en la Casa legislativa, aún mantiene como primer ítem la prohibición de cualquier intento de limitar la libertad de expresión, la libertad de prensa y la libertad del ejercicio del periodismo.

\section{e) Bolivia}

Dentro del grupo de países que pertenecen al MERCOSUL, Bolivia es uno dos que no tiene una ley reglamentando el acceso a la información del Estado. La norma legal más cercana a la temática es la Constitución boliviana que en su artículo 21 declara de manera sucinta el acceso a la información como un derecho civil: "[...] comunicar, informar, opinar, interpretar y acceder a la información, de manera individual o colectiva."

Intentando aclarar y reglamentar ese derecho civil el Ministerio de Transparencia y Lucha Contra la Corrupción de Bolivia estructuró un primer esbozo de proyecto de ley con la finalidad de garantizar la transparencia y el acceso a la información pública. Fruto de los esfuerzos del Ministerio fue presentado el Proyecto de Ley 404/2013 que en 47 artículos busca colocar a Bolivia en el mapa de los países que tienen una ley de acceso a la información.

Por tratarse de un proyecto en fase inicial, aún hay muchas discusiones en torno al texto y de las delimitaciones del proyecto. Prueba de eso es que desde agosto de 2013 se realizaron audiencias públicas para que sea posible escuchar y debatir el texto con varios segmentos de la sociedad. Según Romero (2013)

El Proyecto de Ley 404/2013-2014 posee 47 artículos, fue trabajado por el Ministerio de Transparencia y Lucha Contra la Corrupción y tiene el objetivo de promover la transparencia, el correcto manejo y administración de los recursos públicos y del Estado Plurinacional. De este modo, cumplir el mandato Constitucional que, en su artículo 21, establece como un derecho civil acceder a la información pública, interpretarla, analizarla y comunicarla libremente de manera individual o colectiva.

\section{f) Colombia}

La actual Ley de Transparencia y del Derecho de Acceso a la Información Pública de Colombia, Ley no 1.712/2014 fue promulgada en marzo y es una evolución de la Ley $n^{0}$ 57/1985. Se hizo necesaria hacer una revisión debido a la dinámica en el entorno de la información, especialmente por asuntos de carácter tecnológico y para contemplar los principios de acceso y transparencia emanados de la Constitución de 1991, especialmente en los artículos 15 y 20.

ARTíCULO 15. Todas las personas tienen derecho a su intimidad personal y familiar y a su buen nombre, y el Estado debe respetarlos y hacerlos respetar. De igual modo, tienen derecho a conocer, actualizar y rectificar las informaciones que se hayan recogido sobre ellas en bancos de datos y en archivos de entidades públicas y privadas. En la recolección, tratamiento y circulación de datos se respetarán la libertad y demás garantías consagradas en la Constitución. 
La correspondencia y demás formas de comunicación privada son inviolables. Sólo pueden ser interceptadas o registradas mediante orden judicial, en los casos y con las formalidades que establezca la ley.

Para efectos tributarios o judiciales y para los casos de inspección, vigilancia e intervención del Estado podrá exigirse la presentación de libros de contabilidad y demás documentos privados, en los términos que señale la ley.

$[\ldots]$

ARTICULO 20. Se garantiza a toda persona la libertad de expresar y difundir su pensamiento y opiniones, la de informar y recibir información veraz e imparcial, y la de fundar medios masivos de comunicación.

Estos son libres y tienen responsabilidad social. Se garantiza el derecho a la rectificación en condiciones de equidad. No habrá censura. (COLOMBIA. CONSTITUCIÓN, 1991)

\section{g) Ecuador}

Considerando varios principios, dentro de ellos el artículo 19 de la Constitución Política de la República y el Pacto Internacional de Derechos Civiles y Políticos, y además la Convención Interamericana de Derechos Humanos, Ecuador promulgó, el 18 de abril de 2004, la Ley Orgánica de Transparencia y Acceso a La Información Pública (LOTAIP). Basado en el principio de la publicidad de la información pública, conforme su artículo1ํㅡㄹ

El acceso a la información pública es un derecho de las personas que garantiza el Estado. Toda la información que emane o que esté en poder de las instituciones, organismos y entidades, personas jurídicas de derecho público o privado que, para el tema materia de la información tengan participación del Estado o sean concesionarios de éste, en cualquiera de sus modalidades, conforme lo dispone la Ley Orgánica de la Contraloría General del Estado; las organizaciones de trabajadores y servidores de las instituciones del Estado, instituciones de educación superior que perciban rentas del Estado, las denominadas organizaciones no gubernamentales (ONG's), están sometidas al principio de publicidad; por lo tanto, toda información que posean es pública, salvo las excepciones establecidas en esta Ley. (LOTAIP, 2004)

A pesar de las garantías contenidas en la LOTAIP, la Sociedad Interamericana de Prensa, en la Asamblea de 2013 en Denver, considera que la Ley continúa sin ser aplicada, haciendo al país inoperante en relación al acceso a la información.

\section{h) Perú}

Desarrollada a partir del inciso $\mathrm{V}$ del artículo $2^{\circ}$ de la Constitución Política del Perú, la Ley 27.806 (Ley de Transparencia y Acceso a la Información Pública) fue promulgada en 2002 y rectifica el derecho individual de "solicitar sin expresión de causa la información que requiera y a recibirla de cualquier entidad pública, en el plazo legal, con el costo que suponga el pedido" (PERÚ. CONSTITUCIÓN, 1993).

Modificada en febrero de 2003 por la Ley 27.927, con el fin de proporcionar más transparencia, la Ley 27.806/2002 tiene como principio fundamental la publicidad de las acciones y disposiciones de la administración pública peruana.

\section{i) Uruguay}

Dentro del grupo de países fundadores del MERCOSUR, Uruguay fue la primera nación a promulgar y a reglamentar una Ley de Acceso a la Información de alcance irrestricto a todos los entes de la administración pública estatal o no estatal, lo que ocurrió en 2008 en la gestión del entonces presidente Tabaré Vázquez. La Ley 18.831 estableció el siguiente objeto:

Artículo 1ํ- (Objeto de la ley) - La presente ley tiene por objeto promover la transparencia de la función administrativa de todo organismo público, sea o no estatal, y garantizar el derecho fundamental de las personas al acceso a la información pública (URUGUAY, 2008). 
La reglamentación de la ley ocurrió en 2010, con el Decreto oㅜ232/2010, del 2 de agosto de 2010, que, entre otras disposiciones, especifica las reglas para la clasificación de documentos como confidenciales o parcialmente restringidos, ya introducidas por la ley. El decreto también expone los principios orientadores del acceso a la información pública en Uruguay: libertad de información, transparencia, publicidad máxima, divisibilidad, ausencia de ritualismos, la no discriminación, oportunidad, responsabilidad y gratuidad (URUGUAY, 2010).

\section{j) Venezuela}

Una de las críticas recurrentes al gobierno del presidente Hugo Chávez (1999-2013) fueron motivadas por la censura impetrada contra los medios de prensa y al control de informaciones procedentes de la administración pública. La verdad es que además de no tener una ley de acceso a la información, actualmente también no existe en el parlamento venezolano proyecto de ley que regule tal acceso. Aunque la Constitución vigente, promulgada en 1999, es un tanto imprecisa cuanto al acceso de los ciudadanos a las informaciones públicas. Y parece no haber en el gobierno sucesor, Nicolás Maduro, ninguna indicación en el sentido de promover la libertad de acceso a la información en Venezuela, al igual que los socios del Mercosur.

\section{Transparencia y sigilo}

\subsection{Transparencia}

Según el diccionario Michaelis (2004, p. 2101) ser transparente es el acto de ser claro, franco, que permite ser conocido y que "deja observar un sentido oculto". El movimiento de transparencia inició fuertemente en el año 2000, actualmente es la fuerza motivadora de la sociedad para el combate a la corrupción. El principal factor garantizador de transparencia es el acceso a la información. Para Mendel (2009, p. 23) la necesidad del acceso a la información sobre el control de los organismos públicos es la principal herramienta de combate a la corrupción. Dividido en dos etapas, transparencia activa y pasiva, el acceso a las informaciones gubernamentales se configura como el núcleo de las leyes de acceso a la información.

\section{a) Transparencia Activa}

La transparencia activa puede ser el motor principal del acceso a la información y, por ser elemento de primera grandeza en el campo de la libertad de acceso a la información, es el tópico de las normas que tratan de la materia que se alinea directamente más a la democratización del mecanismo público.

De los siete países que tienen leyes de acceso, incluyendo Brasil, todos los documentos presuponen la transparencia activa, que puede ser definida como la iniciativa del propio Estado para divulgar informaciones de interés general, sin que haya previa solicitud (EIRÃO, SILVA, CAVALCANTE, 2014). Este hecho revela la importancia de ese aspecto en los procesos de libertad de acceso y democracia.

Hay casos en que la transparencia activa está claramente definida y las directrices para su aplicación se explican en el dispositivo legal. A veces manifestada literalmente, a ejemplo de los textos de Chile y de Colombia, la transparencia activa, o transparencia proactiva, puede ser expresada en los textos legales con el concepto de "información mínima" o "divulgación de informaciones independiente de requerimiento". Este último caso se refiere a las normas de: Argentina, Brasil, Ecuador, Perú y Uruguay. El artículo $3^{\underline{a}}$ de la Ley 1.712/2014, de Colombia, aclara el principio de la transparencia activa para el cual:

El derecho de acceso a la información no radica únicamente en la obligación de dar respuesta a las peticiones de la sociedad, sino también en el deber de los sujetos obligados de promover y generar una cultura de transparencia, lo que conlleva la obligación de publicar y divulgar documentos y archivos que plasman la actividad estatal y de interés público, de forma rutinaria y 
proactiva, actualizada, accesible comprensible, atendiendo a límites razonables del talento humano y recursos físicos y financieros. (COLOMBIA, 2014)

Por otra parte, los dos proyectos de ley, Bolivia y Paraguay, también externan la necesidad de que los portales web de los gobiernos deben presentar determinado contenido mínimo sobre acciones gubernamentales.

La transparencia activa es el

[...] tipo más deseable de transparencia pública y denota la preocupación del Estado en dar publicidad a sus acciones, suministrando insumos para que el ciudadano pueda monitorear los rumbos de la Administración. (EIRÃO, SILVA, CAVALCANTE, 2013)

Y si la existencia de las leyes de acceso a la información en los países estudiados significa cambio de paradigma y de actitud por parte de los Estados y de las sociedades, se puede decir que el principio de la transparencia activa es la representación más exacta de la democratización de la información que es, compulsoriamente, divulgada en los portales gubernamentales. Además de eso, reitera la importancia de la web como medio para esa democratización.

\section{b) Transparencia Pasiva}

La transparencia pasiva involucra a toda la obligación del Estado en atender una determinada demanda de información requerida por la sociedad. Diferentemente de la transparencia activa, la transparencia pasiva siempre es reactiva y envuelve procesos más complejos de servicio, sea por la naturaleza de la demanda o por el esfuerzo emprendido para o atención. Dadas estas complejidades, los diplomas legales explican todas las condiciones, exigencias y términos que una información sea solicitada.

De los diez países estudiados, siete tienen leyes de acceso a la información (Argentina, Brasil, Chile, Colombia, Ecuador, Perú, Uruguay) sólo Perú y Colombia no enumeran las reglas básicas para la solicitud de información, esos países apenas garantizan que todas las personas físicas y jurídicas tienen el derecho de exigir información. Venezuela por no tener una ley específica, no presenta condición para demanda de información. Por otro lado, Paraguay y Bolivia, tienen proyectos en tramitación en sus casas legislativas, a continuación las otras cinco leyes que describen las reglas básicas para la solicitud de información:

1. Identificación del solicitante (nombre, profesión, dirección);

2. Descripción clara de la información requerida;

3. Formas de entrega de la información (físico, digital, email, fax);

En las leyes y proyectos investigados existe alguna unanimidad en relación a:

- Declaración de la gratuidad de la solicitud de información;

- Las únicas tasas posibles de cobranza se refieren a los gastos de envío de los documentos solicitados;

- Preferencia por la atención inmediata de la demanda, excepto cuando se trata de una información confidencial o reservada que obedecerá a reglas específicas.

- Solicitud sólo por escrito, excepto la ley colombiana y el proyecto de ley paraguayo y boliviano que permiten también por vía oral;

- Instancias de recursos para denegatorias de información;

- Imputación legal y administrativa a los agentes que nieguen acceso a la solicitación.

Cuando el organismo no atienda rápidamente determinado requerimiento de información, cada país estipuló plazos mínimos para la respuesta referente a la solicitud: 
Cuadro 2: Plazos mínimos para respuesta a la solicitación de información

\begin{tabular}{|l|l|}
\hline \multicolumn{1}{|c|}{ País } & \multicolumn{1}{c|}{ Plazo } \\
\hline Argentina & 10 días + 10 días (prorrogación) \\
\hline Brasil & 20 días + 10 días (prorrogación) \\
\hline Bolivia & 10 días + 20 días (prorrogación) \\
\hline Chile & 20 días \\
\hline Colombia & No declara \\
\hline Ecuador & 10 días + 5 días (prorrogación) \\
\hline Paraguay & 10 días + 10 días (prorrogación) \\
\hline Perú & 7 días + 5 días (prorrogación) \\
\hline Uruguay & 20 días \\
\hline
\end{tabular}

Fuente: elaboración de los autores.

Terminados esos plazos, cada organismo debe manifestarse sobre la posibilidad de atender o no la demanda. Las siete leyes y los dos proyectos, determinan que la denegatoria de información, parcial o total, deberá ser hecha por escrito y con fundamento legal que explique los motivos de no atender a la demanda, caso que sólo podrá ocurrir cuando se trate de información sigilosa o de acceso reservado.

\subsection{Sigilo}

Uno de los temas más delicados cuando se habla en acceso a la información pública es lo que dice respecto a informaciones sigilosas, una vez que, en este caso, dos intereses pueden divergir: el deseo de los ciudadanos en conocer milimétricamente las acciones ejercidas por la entidad pública y la necesidad del Estado de resguardar informaciones que pueden colocarlo en riesgo o exponer a los propios ciudadanos.

En este sentido, una de las finalidades de las leyes de acceso a la información es exactamente funcionar como mediadora de los grados de sigilo de documentos al considerar la condición de sigilo como excepción, evitando la restricción de informaciones de real interés para la sociedad. En el ámbito del MERCOSUR, el grado de sigilo se configura como una temática bastante relevante delante del pasado reciente de muchos de sus componentes, marcados por regímenes autoritarios.

De hecho, a excepción de Paraguay, que no trata de sigilo en su proyecto de ley de acceso a la información, todas las leyes, decretos y proyectos analizados presentan informaciones sigilosas como excepción. Hay gran semejanza entre asuntos que pueden ser considerados como confidenciales, de los que se destacan: la defensa nacional; la seguridad pública; transacciones económicas y comerciales internacionales; el sistema financiero nacional; la seguridad y la integridad física de los ciudadanos. Mientras tanto, se destaca, que no todos los países previnieron en sus leyes todos los temas que pueden determinar la necesidad de sigilo. Es el caso de Ecuador que, además de la defensa nacional, dejó para leyes específicas el establecimiento de informaciones que pueden suscitar sigilo al ser clasificadas como reservadas. Se trata de una medida cuestionable, teniendo en vista que la razonabilidad de que la ley general pronostique todos los casos.

Sobre la clasificación de las informaciones sigilosas también se puede afirmar que hay similitudes entre las legislaciones, llegando a tres categorías: información reservada, secreta y confidencial. En general, la información reservada es la que se refiere a la defensa nacional, la temática más relevante, siendo también la categoría con mayor plazo de protección.

En relación a los plazos de sigilo, no hubo mucha unidad entre los países. Hay variaciones relacionadas a las categorías de informaciones sigilosas, bien como el límite máximo de años en que las informaciones pueden estar fuera del alcance del público. Mientras que en Perú las informaciones secretas pueden ser solicitadas cinco años 
después de su clasificación, en Brasil, aquellas clasificadas como ultra secretas están indisponibles por 25 años. Mientras tanto, hay que destacar, dispositivos dejados por las leyes para embargar el acceso a la información con plazo de reserva expirado, en caso que se considere que los datos a ser divulgados podrán traer situaciones de riesgo. Por ejemplo, es el caso de la ley peruana.

\section{Conclusión}

Los resultados obtenidos en el estudio permiten concluir que las Leyes guardan alguna semejanza entre si y que los tres aspectos estudiados encuentran resonancia en mayor o menor grado, en las diferentes leyes. Lógicamente que la aplicación de las leyes puede divergir, dependiendo de la orientación política vigente en cada país, sin embargo es posible vislumbrar influencias de las leyes más antiguas en las leyes creadas recientemente. Esto puede ser verificado principalmente en los criterios establecidos de transparencia pasiva en lo que concierne a las formas del ciudadano efectuar una demanda de información, como en los plazos establecidos de respuestas. En relación a esos aspectos hay, inclusive, mucha semejanza en la redacción de los textos.

A pesar de no haber una interconexión entre los países del MERCOSUR en las políticas de acceso a la información, se verifica que esa interacción fue realizada directamente en los textos legales, una vez que el estudio demuestra que algunas leyes sirven de inspiración a otras, dada la proximidad de los textos.

A pesar de que las leyes de acceso a la información han sido creadas con la intención de mejorar la transparencia de disminuir el sigilo de las acciones del Estado, hay una lucha constante entre transparencia y opacidad. Es evidente que las leyes son avances democráticos, sin embargo, no se puede afirmar el grado de aplicabilidad y sus beneficios reales para los ciudadanos de los países del MERCOSUR.

\section{Notas}

${ }^{1}$ Texto original del proyecto de ley disponible en:

http://www.senado.cl/appsenado/index.php?mo=tramitacion\&ac=getDocto\&iddocto=4166\&tipodoc=mensaje mocion

${ }^{2}$ Disponible en: http://www.leychile.cl/Navegar?idNorma=276363

${ }^{3}$ Detalles del proyecto en:

http://sil2py.senado.gov.py/formulario/VerDetalleTramitacion.pmf?q=VerDetalleTramitacion\%2F101629

\section{Referencias}

ARGENTINA. Decreto n. 1.172, de 3 de deciembro de 2003. Apruébanse los Reglamentos Generales de Audiencias Públicas para el Poder Ejecutivo Nacional, para la Publicidad de la Gestión de Intereses en el ámbito del Poder Ejecutivo Nacional, para la Elaboración Participativa de Normas, del Acceso a la Información Pública para el Poder Ejecutivo Nacional y de Reuniones Abiertas de los Entes Reguladores de los Servicios Públicos, Formularios de inscripciones, registro y presentación de opiniones y propuestas. Establécese el acceso libre y gratuito vía Internet a la edición diaria del Boletín Oficial de la República Argentina. InfoLEG, Buenos Aires. Disponible en: <http://infoleg.gov.ar/infoleglnternet/anexos/90000-94999/90763/norma.htm>. Acceso: 18 junio 2014.

BOLIVIA. Constitución (2007). Constitución Política del Estado. Disponible en: <http://www.patrianueva.bo/constitucion/>. Acceso: 24 junio 2014).

BRASIL. Constitución (1988). Constituição da Republica Federativa do Brasil. Disponible en: <http://www2.camara.leg.br/atividadelegislativa/legislacao/Constituicoes Brasileiras/constituica01988.html/ConstituicaoTextoAtualizado EC81.pdf>. Acceso: 18 junio 2014).

CHILE. Constitución (1980). Constitución política de la república de Chile. Disponible en: <http://www. leychile.cl/Navegar?idNorma=242302>. Acceso: 24 junio 2014).

COLOMBIA. Constitución (1993). Constitución Política de Colombia. Disponible en: <http://www.constitucioncolombia.com/>. Acceso: 24 junio 2014.

EIRÃO, Thiago Gomes; SILVA, Terezinha Elisabeth da; CAVALCANTE, Raphael da Silva Cavalcante. Lei de Acesso à Informação (LAl): investigação da transparência ativa nos três poderes. E-legis: Revista Eletrônica do Programa de Pós-Graduação da 


\section{Ð10 Relacionando la legislación sobre acceso a la Información ...}

Câmara dos Deputados, Brasília, n. 13, p. 43-56 Disponible en: <http://elegisbr.com/cefor/index.php/e-legis/article/view/160/204>. Acceso: 24 junio 2014.

FERNÁNDEZ, Hernán Larraín; MUJICA, Jaime Gazmuri. Proyecto de ley sobre acceso a la informacion pública. Disponible en: $<$ http://www. senado.cl/appsenado/index.php?mo=tramitacion\&ac=getDocto\&iddocto=4166\&tipodoc=mensaje mocion >. Acceso: 24 junio 2014.

LEY Orgánica de Transparencia y Acceso a La Información Pública. No. 24, publicado en el Registro Oficial Suplemento 337 del 18 de Mayo del 2004. Quito: Congreso Nacional.

MENDEL, Toby. Liberdade de informação: um estudo de direito comparado. Disponible en: $<$ http://www.acessoainformacao.gov.br/central-de-conteudo/publicacoes/liberdade-informacao-estudo-direito-comparadounesco.pdf>. Acceso: 24 junio 2014.

MICHAELIS: moderno dicionário da língua portuguesa. São Paulo: Melhoramentos, 2004. 980 p.

PARAGUAY. Constitución (1992). Constitución de la Republica del Paraguay. Disponible en: $<$ http://www.tsje.gov.py/constituciones.php>. Acceso 24 junio 2014.

PERÚ. Constitución (1993). Constitución Política del Perú. Disponible en: <http://www.tc.gob.pe/legconperu/constitucion.html>. Acceso: 24 junio 2014.

ROMERO, Raúl Morales. Análisis de ley de transparencia y acceso a la información. La Razón, la gaceta jurídica, 13 agosto 2014. Disponible en: <http://www.la-razon.com/suplementos/la gaceta juridica/Analisis-Ley-Transparencia-AccesoInformacion 0 1886811386.html>. Acceso: 24 junio 2014.

SOCIEDADE Intermericana de Imprensa. Acesso à informação. 2013. Disponible en: <http://www. sipiapa.org/ptbr/asamblea/acesso-a-informacao-6/>. Acceso: 10 junio 2014.

URUGUAY. Ley n. 18.831, de 17 de octubre de 2008. AGESIC, Montevideo. Disponible en: <http://www.agesic.gub.uy/innovaportal/v/161/1/agesic/ley n\%C2\%BA 18381.html>. Acceso: 20 junio 2014.

URUGUAY. Decreto n. 232, de 3 de agosto de 2010. AGESIC, Montevideo. Disponible en: <http://www.agesic.gub.uy/innovaportal/v/1121/1/agesic/decreto n\%C2\%B0 232010 de 2 de agosto de 2010.html>. Acceso: 18 junio 2014. 


\section{Datos de los autores}

Terezinha Elisabeth da Silva

Grado post-doctoral en Ciencia de la Información por la Universidad Federal de Minas Gerais, doctora en Multimedia, Maestría en Estudios de Bibliotecología, graduada em Bibliotecología. Analista legislativo de la Cámara de Diputados de Brasil y la enseñanza del Curso de Master Profesional en Gestión de la Información. terezinha.elisabeth.silva@gmail.com

\section{Thiago Gomes Eirão}

Candidato a Doctor en Ciencia de la Información por la Universidad de Brasilia. Maestría en Ciencias de la Información por la Universidad de Brasilia, graduado en bibliotecología. Analista legislativo de la Cámara de Diputados de Brasil.

thiagoe@gmail.com

\section{Raphael da Silva Cavalcante}

Magister en Ciencia de la Información por la Universidad de Brasilia, graduado en Bibliotecología. Analista legislativo de la Cámara de Diputados de Brasil.

xrapha@gmail.com

Recibido-Received : 2014-08-18
Aceptado-Accepted : 2014-09-30

\section{(cc) $\mathbf{E Y}$}

This work is licensed under a Creative Commons Attribution 4.0

United States License.

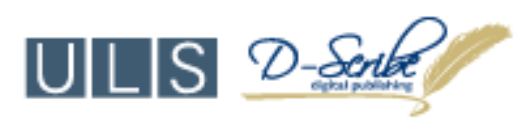

This journal is published by the University Library System of the University of Pittsburgh as part of its D-Scribe Digital Publishing Program and is cosponsored by the University of Pittsburgh Press. 